JOURNAL OF THE

AMERICAN MATHEMATICAL SOCIETY

Volume 13, Number 2, Pages 333-341

S 0894-0347(99)00323-9

Article electronically published on October 25, 1999

\title{
REAL RATIONAL CURVES IN GRASSMANNIANS
}

\author{
FRANK SOTTILE
}

\section{INTRODUCTION}

Fulton asked how many solutions to a problem of enumerative geometry can be real, when that problem is one of counting geometric figures of some kind having specified position with respect to some given general figures [5]. For the problem of plane conics tangent to five general (real) conics, the surprising answer is that all 3264 may be real [13]. Similarly, given any problem of enumerating $p$-planes incident on some given general subspaces, there are real subspaces such that each of the (finitely many) incident $p$-planes is real [17]. We show that the problem of enumerating parameterized rational curves in a Grassmannian satisfying simple (codimension 1) conditions may have all of its solutions real.

This problem of enumerating rational curves in a Grassmannian arose in at least two distinct areas of mathematics. The number of such curves was predicted by the formula of Vafa and Intriligator [20, 8] from mathematical physics. It is also the number of complex dynamic compensators which stabilize a given linear system, and the enumeration was solved in this context [12 11]. The question of real solutions also arose in systems theory [3. This application will be discussed in Section 4.

\section{Statement of Results}

Fix integers $m, p>1$ and $q \geq 0$. Set $n:=m+p$. Let $\mathbf{G}$ be the Grassmannian of $p$-planes in $\mathbb{C}^{n}$. The space $\mathcal{M}_{q}$ of maps $M: \mathbb{P}^{1} \rightarrow \mathbf{G}$ of degree $q$ has dimension $N:=p m+q n$ 4 19]. If $L$ is an $m$-plane and $s \in \mathbb{P}^{1}$, then the collection of all maps $M \in \mathcal{M}_{q}$ satisfying $M(s) \cap L \neq\{0\}$ is an irreducible subvariety of codimension 1 . Consider the following enumerative problem.

Given general points $s_{1}, \ldots, s_{N}$ in $\mathbb{P}^{1}$ and general $m$-planes $L_{1}, \ldots, L_{N}$ in $\mathbb{C}^{n}$, how many maps $M \in \mathcal{M}_{q}$ satisfy $M\left(s_{i}\right) \cap L_{i} \neq\{0\}$ for $i=1, \ldots, N$ ?

Since $\mathbf{G}$ is a homogeneous space, Kleiman's Theorem [9] shows there are finitely many solutions and no multiplicities.

Rosenthal [14] interpreted the solutions as a linear section of a projective embedding of $\mathcal{M}_{q}$, and Ravi, Rosenthal, and Wang [12, 11] gave a formula for the degree $d$ of its closure $\mathcal{K}_{q}$ in this embedding. Thus the number of solutions (counted with multiplicity) is at most $d$. The difference between $d$ and the number of solutions counts points common to both the linear section and to the boundary $\mathcal{K}_{q}-\mathcal{M}_{q}$ of $\mathcal{K}_{q}$.

Received by the editors April 29, 1999 and, in revised form, August 24, 1999. 2000 Mathematics Subject Classification. Primary 14M15, 14N35, 14P99, 65H20, 93 B55. Research at MSRI supported in part by NSF grant DMS-9701755. 
Bertram [1] studied this and other intersection problems on $\mathcal{M}_{q}$ using a different compactification $\mathcal{Q}_{q}$ of $\mathcal{M}_{q}$. He used an explicit moving lemma to show there are finitely many solutions to these problems on $\mathcal{Q}_{q}$ with none in the boundary $\mathcal{Q}_{q}-\mathcal{M}_{q}$ of $\mathcal{Q}_{q}$. He also determined the small quantum cohomology ring of $\mathbf{G}$, which gives formulas for these intersection numbers. For our problem (1.1), the formula of Bertram coincides with the formula of Ravi, Rosenthal, and Wang. This shows there are no points common to both the linear section and to the boundary of $\mathcal{K}_{q}$, and so there are $d$ solutions to (1.1).

When the $s_{i}$ and $L_{i}$ are real, there may be fewer than $d$ real solutions. We show there are real $s_{i}$ and $L_{i}$ such that each of the $d$ solutions are real.

Theorem 1.1. There exist $m$-planes $L_{1}, \ldots, L_{N}$ in $\mathbb{R}^{n}$ and points $s_{1}, \ldots, s_{N} \in \mathbb{P}_{\mathbb{R}}^{1}$ so that there are exactly $d$ maps $M: \mathbb{P}^{1} \rightarrow \mathbf{G}$ of degree $q$ which satisfy $M\left(s_{i}\right) \cap L_{i} \neq$ $\{0\}$ for each $i=1, \ldots, N$, and each of these are real.

Our proof is elementary in that it argues from the equations for the locus of maps $M$ which satisfy $M(s) \cap L \neq\{0\}$. A consequence is that we obtain fairly explicit choices of $s_{i}$ and $L_{i}$ that give only real maps, which we discuss in Section 4 . Our proof uses neither Kleiman's Theorem nor Bertram's Moving Lemma, and thus it provides a new and elementary proof that there are $d$ solutions to the enumerative problem (1.1).

\section{The quantum Grassmannian}

The space $\mathcal{M}_{q}$ of maps $\mathbb{P}^{1} \rightarrow \mathbf{G}$ of degree $q$ is a smooth quasi-projective algebraic variety. A smooth compactification is provided by a quot scheme $\mathcal{Q}_{q}$ [19]. By definition, there is a universal exact sequence

$$
0 \rightarrow \mathcal{S} \rightarrow \mathbb{C}^{n} \otimes \mathcal{O}_{\mathbb{P}^{1} \times \mathcal{Q}_{q-1}} \rightarrow \mathcal{T} \rightarrow 0
$$

of sheaves on $\mathbb{P}^{1} \times \mathcal{Q}_{q}$ where $\mathcal{S}$ is a vector bundle of degree $-q$ and rank $p$. Twisting the determinant of $\mathcal{S}$ by $\mathcal{O}_{\mathbb{P}^{1}}(q)$ and pushing forward to $\mathcal{Q}_{q}$ induces a Plücker map

$$
\mathcal{Q}_{q} \rightarrow \mathbb{P}\left(\bigwedge^{p} \mathbb{C}^{n} \otimes H^{0}\left(\mathcal{O}_{\mathbb{P}^{1}}(q)\right)^{*}\right)
$$

which is the analog of the Plücker embedding of $\mathbf{G}$. The Plücker map is an embedding of $\mathcal{M}_{q}$, and so its image $\mathcal{K}_{q}$ provides a different compactification of $\mathcal{M}_{q}$. We call $\mathcal{K}_{q}$ the quantum Grassmannian. (This space is called the Uhlenbeck compactification in 22].) Our proof of Theorem 1.1] exploits some of its structures that were elucidated in systems theory.

The Plücker map fails to be injective on the boundary $\mathcal{Q}_{q}-\mathcal{M}_{q}$ of $\mathcal{Q}_{q}$. Indeed, Bertram [1] constructs a $\mathbb{P}^{p-1}$ bundle over $\mathbb{P}^{1} \times \mathcal{Q}_{q-1}$ that maps onto the boundary of $\mathcal{Q}_{q}$, with its restriction over $\mathbb{P}^{1} \times \mathcal{M}_{q-1}$ an embedding. On this projective bundle, the Plücker map factors through the base $\mathbb{P}^{1} \times \mathcal{Q}_{q-1}$ and the image of a point in the base is $s \cdot S$, where $s$ is the section of $\mathcal{O}_{\mathbb{P}^{1}}(1)$ vanishing at $s \in \mathbb{P}^{1}$ and $S$ is the image of a point in $\mathcal{Q}_{q-1}$ under its Plücker map. This identifies the image of the exceptional locus of the Plücker map with the image of $\mathbb{P}^{1} \times \mathcal{K}_{q-1}$ in $\mathcal{K}_{q}$ under a map $\pi$ which is given in (2.1) below.

More concretely, a point in $\mathcal{Q}_{q}$ may be (non-uniquely) represented by a $p \times n$ matrix $M$ of forms in $s, t$ with homogeneous rows and whose maximal minors have degree $q[10]$. The image of such a point under the Plücker map is the collection 
of maximal minors of $M$. The maps in $\mathcal{M}_{q}$ are represented by matrices whose maximal minors have no common factors: Given such a matrix $M$, the association

$$
\mathbb{P}^{1} \ni(s, t) \longmapsto \text { row space } M(s, t)
$$

defines a map of degree $q$.

The collection $\left(\begin{array}{c}{[n]} \\ p\end{array}\right)$ of $p$-subsets of $\{1, \ldots, n\}$ index the maximal minors of $M$. For $\alpha \in\left(\begin{array}{c}{[n]} \\ p\end{array}\right)$ and $0 \leq a \leq q$, let $z_{\alpha^{(a)}}$ be the coefficient of $s^{a} t^{q-a}$ in the $\alpha$ th maximal minor of $M$. These $z_{\alpha^{(a)}}$ provide Plücker coordinates for the space $\mathbb{P}\left(\bigwedge^{p} \mathbb{C}^{n} \otimes H^{0}\left(\mathcal{O}_{\mathbb{P}^{1}}(q)\right)^{*}\right)$. Let $\mathcal{C}_{q}:=\left\{\alpha^{(a)}: \alpha \in\left(\begin{array}{c}{[n]} \\ p\end{array}\right), 0 \leq a \leq q\right\}$ be the indices of these Plücker coordinates. Then the image of the exceptional locus in $\mathcal{K}_{q}$ is the image of the map $\pi: \mathbb{P}^{1} \times \mathcal{K}_{q-1} \rightarrow \mathcal{K}_{q}$ defined by

$$
\pi:\left([A, B],\left(x_{\beta^{(b)}}: \beta^{(b)} \in \mathcal{C}_{q-1}\right)\right) \longmapsto\left(A x_{\alpha^{(a)}}-B x_{\alpha^{(a-1)}}: \alpha^{(a)} \in \mathcal{C}_{q}\right),
$$

where $x_{\alpha^{(q)}}=x_{\alpha^{(-1)}}=0$.

The relevance of the quantum Grassmannian $\mathcal{K}_{q}$ to the enumerative problem (1.1) is seen by considering the condition for a map $M \in \mathcal{M}_{q}$ to satisfy $M(s, t) \cap L \neq\{0\}$ where $L$ is an $m$-plane in $\mathbb{C}^{n}$ and $(s, t) \in \mathbb{P}^{1}$. If we represent $L$ as the row space of an $m \times n$ matrix, also written $L$, then this condition is

$$
0=\operatorname{det}\left[\begin{array}{c}
L \\
M(s, t)
\end{array}\right]=\sum_{\alpha \in\left(\begin{array}{c}
{[n]} \\
p
\end{array}\right)} f_{\alpha}(s, t) l_{\alpha},
$$

the second expression given by Laplace expansion of the determinant along the rows of $M$. Here, $l_{\alpha}$ is the appropriately signed maximal minor of $L$. If we expand the forms $f_{\alpha}(s, t)$ in this last expression, then we obtain

$$
\sum_{\alpha^{(a)} \in \mathcal{C}_{q}} z_{\alpha^{(a)}} s^{a} t^{q-a} l_{\alpha}=0,
$$

a linear equation in the Plücker coordinates of $M$. Thus the solutions $M \in \mathcal{M}_{q}$ to the enumerative problem (1.1) are a linear section of $\mathcal{M}_{q}$ in its Plücker embedding, and so the degree $d$ of $\mathcal{K}_{q}$ provides an upper bound on the number of solutions.

The set $\mathcal{C}_{q}$ of Plücker coordinates has a natural partial order

$$
\alpha^{(a)} \leq \beta^{(b)} \Longleftrightarrow a \leq b \text { and } \alpha_{i} \leq \beta_{b-a+i} \text { for } i=1,2, \ldots, p-b+a .
$$

The poset $\mathcal{C}_{q}$ is graded with the rank, $\left|\alpha^{(a)}\right|$, of $\alpha^{(a)}$ equal to an $+\sum_{i} \alpha_{i}-i$. It is also a distributive lattice. Figure $\square$ shows $\mathcal{C}_{1}$ when $p=2$ and $m=3$. Given $\alpha^{(a)} \in \mathcal{C}_{q}$, define the quantum Schubert variety

$$
Z_{\alpha^{(a)}}:=\left\{\left(z_{\beta^{(b)}}\right) \in \mathcal{K}_{q}: z_{\beta^{(b)}}=0 \text { if } \beta^{(b)} \not \leq \alpha^{(a)}\right\} .
$$

Let $\mathcal{H}_{\alpha^{(a)}}$ be the hyperplane defined by $z_{\alpha^{(a)}}=0$. The main technical result we use is the following.

Proposition 2.1 ([11, 12]). Let $\alpha^{(a)} \in \mathcal{C}_{q}$. Then

(i) $Z_{\alpha^{(a)}}$ is an irreducible subvariety of $\mathcal{K}_{q}$ of dimension $\left|\alpha^{(a)}\right|$.

(ii) The intersection of $Z_{\alpha^{(a)}}$ and $\mathcal{H}_{\alpha^{(a)}}$ is generically transverse and we have

$$
Z_{\alpha^{(a)}} \cap \mathcal{H}_{\alpha^{(a)}}=\bigcup_{\beta^{(b)} \lessdot \alpha^{(a)}} Z_{\beta^{(b)}}
$$




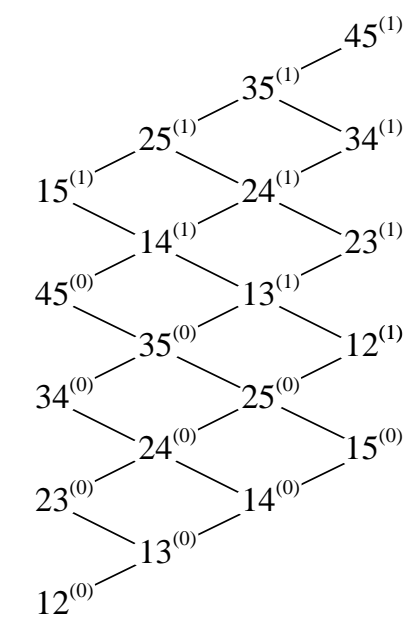

FiguRE $1 . \mathcal{C}_{1}$ with $p=2$ and $m=3$.

Another proof of (ii) is given in [18, which shows that (ii) is a scheme-theoretic equality. From (ii) and Bézout's theorem, we obtain the following recursive formula for the degree of $Z_{\alpha^{(a)}}$ :

$$
\operatorname{deg} Z_{\alpha^{(a)}}=\sum_{\beta^{(b)} \lessdot \alpha^{(a)}} \operatorname{deg} Z_{\beta^{(b)}} .
$$

Since the minimal quantum Schubert variety is a point, we deduce the formula of 12 .

Corollary 2.2. The degree $d$ of $\mathcal{K}_{q}$ is the number of maximal chains in the poset $\mathcal{C}_{q}$.

For example, when $p=2$ and $m=3$, the degree of $\mathcal{K}_{1}$ is 55 .

An alternative proof of Corollary [2.2 is given in [18] by explicitly deforming $\mathcal{K}_{q}$ to the toric variety associated with the poset $\mathcal{C}_{q}$.

\section{Proof of Theorem 1.1}

Let $L(s, t)$ be the $m$-plane osculating the parameterized rational normal curve

$$
\gamma:(s, t) \in \mathbb{P}^{1} \longmapsto\left(s^{n-1}, t s^{n-2}, \ldots, t^{n-2} s, t^{n-1}\right) \in \mathbb{P}^{n-1}
$$

at the point $\gamma(s, t)$. Then $L(s, t)$ is the row space of the $m \times n$ matrix of forms with rows $\gamma(s, t), \gamma^{\prime}(s, t), \ldots, \gamma^{(m-1)}(s, t)$, the derivative taken with respect to the parameter $t$. Write $L(s, t)$ for this matrix. For $\alpha \in\left(\begin{array}{c}{[n]} \\ p\end{array}\right)$, the maximal minor of $L(s, t)$ complementary to $\alpha$ is $s^{\left(\begin{array}{c}m \\ 2\end{array}\right)} \cdot(-1)^{|\alpha|} l_{\alpha} \cdot s^{|\alpha|} t^{m p-|\alpha|}$, where $|\alpha|:=\sum_{i} \alpha_{i}-i$ and $(-1)^{|\alpha|} l_{\alpha}$ is the corresponding maximal minor of $L(1,1)$. For $(s, t) \in \mathbb{P}^{1}$, let $\mathcal{H}(s, t)$ be the hyperplane given by the linear form

$$
\Lambda(s, t):=\sum_{\alpha^{(a)} \in \mathcal{C}_{q}} z_{\alpha^{(a)}} l_{\alpha} s^{\left|\alpha^{(a)}\right|} t^{N-\left|\alpha^{(a)}\right|} .
$$


Let $M$ be a matrix representing a map in $\mathcal{M}_{q}$. Then

$$
\operatorname{det}\left[\begin{array}{c}
L(s, t) \\
M\left(s^{n}, t^{n}\right)
\end{array}\right]=s^{\left(\begin{array}{c}
m \\
2
\end{array}\right)} \sum_{\alpha^{(a)} \in \mathcal{C}_{q}} z_{\alpha^{(a)}} s^{a n} t^{(q-a) n} l_{\alpha} s^{|\alpha|} t^{m p-|\alpha|}=s^{\left(\begin{array}{c}
m \\
2
\end{array}\right)} \Lambda(s, t) .
$$

Thus $\mathcal{M}_{q} \cap \mathcal{H}(s, t)$ consists of all maps $M: \mathbb{P}^{1} \rightarrow \mathbf{G}$ of degree $q$ which satisfy $M\left(s^{n}, t^{n}\right) \cap L(s, t) \neq\{0\}$.

Theorem [1.1 is a consequence of the following two theorems.

Theorem 3.1. There exist real numbers $s_{1}, \ldots, s_{N}$ such that for any $\alpha^{(a)} \in \mathcal{C}_{q}$ the intersection

$$
Z_{\alpha^{(a)}} \cap \mathcal{H}\left(s_{1}, 1\right) \cap \cdots \cap \mathcal{H}\left(s_{\left|\alpha^{(a)}\right|}, 1\right)
$$

is transverse with all points of intersection real.

Theorem 3.2. If $s_{1}, \ldots, s_{k} \in \mathbb{C}$ are distinct, then for any $\alpha^{(a)} \in \mathcal{C}_{q}$ the intersection

$$
Z_{\alpha^{(a)}} \cap \mathcal{H}\left(s_{1}, 1\right) \cap \cdots \cap \mathcal{H}\left(s_{k}, 1\right)
$$

is proper in that it has dimension $\left|\alpha^{(a)}\right|-k$.

Proof of Theorem 1.1] By Theorem 3.1 there exist real numbers $s_{1}, \ldots, s_{N}$ (necessarily distinct) so that the intersection

$$
\mathcal{K}_{q} \cap \mathcal{H}\left(s_{1}, 1\right) \cap \cdots \cap \mathcal{H}\left(s_{N}, 1\right)
$$

is transverse and consists of exactly $d$ real points. To prove Theorem 1.1, we show that all these points lie in $\mathcal{M}_{q}$. Thus each point in (3.3) represents a real map $M: \mathbb{P}^{1} \rightarrow \mathbf{G}$ of degree $q$ satisfying $M\left(s_{i}^{n}, 1\right) \cap L\left(s_{i}, 1\right) \neq\{0\}$ for $i=1, \ldots, N$.

Let $\pi: \mathbb{P}^{1} \times \mathcal{K}_{q-1} \rightarrow \mathcal{K}_{q}$ be the map (2.1) whose image is the complement of $\mathcal{M}_{q}$ in $\mathcal{K}_{q}$. Then

$$
\begin{aligned}
\pi^{*} \Lambda(s, t) & =\sum_{\alpha^{(a)} \in \mathcal{C}_{q}}\left(A x_{\alpha^{(a)}}-B x_{\alpha^{(a-1)}}\right) l_{\alpha} s^{\left|\alpha^{(a)}\right|} t^{N-\left|\alpha^{(a)}\right|} \\
& =\left(A t^{n}-B s^{n}\right) \sum_{\beta^{(b)} \in \mathcal{C}_{q-1}} x_{\beta^{(b)}} l_{\beta} s^{\left|\beta^{(a)}\right|} t^{N-n-\left|\beta^{(b)}\right|} \\
& =\left(A t^{n}-B s^{n}\right) \Lambda^{\prime}(s, t),
\end{aligned}
$$

where $\Lambda^{\prime}(s, t)$ is the linear form for $\mathcal{K}_{q-1}$ analogous to $\Lambda(s, t)$. Let $\mathcal{H}^{\prime}(s, t)$ be the hyperplane given by the linear form $\Lambda^{\prime}(s, t)$.

Any point in (3.3) but not in $\mathcal{M}_{q}$ is the image of a point $([A, B], x)$ in $\mathbb{P}^{1} \times \mathcal{K}_{q-1}$ satisfying $\pi^{*} \Lambda\left(s_{i}, 1\right)=\left(A-B s_{i}^{n}\right) \Lambda^{\prime}\left(s_{i}, 1\right)$ for each $i=1, \ldots, N$. As the $s_{i}$ are distinct and real, such a point can satisfy $A-B s_{i}^{n}=0$ for at most two $i$. Thus $x \in \mathcal{K}_{q-1}$ lies in at least $N-2$ of the hyperplanes $\mathcal{H}^{\prime}\left(s_{i}, 1\right)$. Since $N-2$ exceeds the dimension $N-n$ of $\mathcal{K}_{q-1}$, there are no such points $x \in \mathcal{K}_{q-1}$, by Theorem 3.2 for maps of degree $q-1$.

Proof of Theorem 3.2, For any $s_{1}, \ldots, s_{k}$, the intersection (3.2) has dimension at least $\left|\alpha^{(a)}\right|-k$. We show it has at most this dimension, if $s_{1}, \ldots, s_{k}$ are distinct.

Suppose $k=\left|\alpha^{(a)}\right|+1$ and let $z \in Z_{\alpha^{(a)}}$. Then $z_{\beta^{(b)}}=0$ if $\beta^{(b)} \not \leq \alpha^{(a)}$ and so the form $\Lambda(s, 1)$ defining $\mathcal{H}(s, 1)$ evaluated at $z$ is

$$
\sum_{\beta^{(b)} \leq \alpha^{(a)}} z_{\beta^{(b)}} l_{\beta} s^{\left|\beta^{(b)}\right|} .
$$


This is a non-zero polynomial in $s$ of degree at most $\left|\alpha^{(a)}\right|$ and thus it vanishes for at most $\left|\alpha^{(a)}\right|$ distinct values of $s$. It follows that (3.2) is empty for $k>\left|\alpha^{(a)}\right|$.

If $k \leq\left|\alpha^{(a)}\right|$ and $s_{1}, \ldots, s_{k}$ are distinct, but (3.2) has dimension exceeding $\left|\alpha^{(a)}\right|-k$, then completing $s_{1}, \ldots, s_{k}$ to a set of distinct numbers $s_{1}, \ldots, s_{\left|\alpha^{(a)}\right|+1}$ would give a non-empty intersection in (3.2), a contradiction.

Proof of Theorem 3.1. We construct the sequence $s_{i}$ inductively. The unique element of rank 1 in $\mathcal{C}_{q}$ is $\alpha^{(0)}$, where $\alpha$ is the sequence $1<2<\cdots<p-1<p+1$. The quantum Schubert variety $Z_{\alpha^{(0)}}$ is a line in Plücker space. Indeed, it is isomorphic to the set of $p$-planes containing a fixed $(p-1)$-plane and lying in a fixed $(p+1)$-plane. By Theorem $3.2 Z_{\alpha^{(0)}} \cap \mathcal{H}(s, 1)$ is then a single, necessarily real, point, for any real number $s$. Let $s_{1}$ be any positive real number.

Suppose we have real numbers $s_{1}, \ldots, s_{k}$ with the property that for any $\beta^{(b)}$ with $\left|\beta^{(b)}\right| \leq k$,

$$
Z_{\beta^{(b)}} \cap \mathcal{H}\left(s_{1}, 1\right) \cap \cdots \cap \mathcal{H}\left(s_{\left|\beta^{(b)}\right|}, 1\right)
$$

is transverse with all points of intersection real.

Let $\alpha^{(a)}$ be an index with $\left|\alpha^{(a)}\right|=k+1$ and consider the 1-parameter family $\mathcal{Z}(s)$ of schemes defined by $Z_{\alpha^{(a)}} \cap \mathcal{H}(s, 1)$. If we restrict the form $\Lambda(s, 1)$ to $z \in Z_{\alpha^{(a)}}$, then we obtain

$$
\sum_{\beta^{(b)} \leq \alpha^{(a)}} z_{\beta^{(b)}} l_{\beta} s^{\left|\beta^{(b)}\right|}
$$

a polynomial in $s$ with leading term $z_{\alpha^{(a)}} s^{\left|\alpha^{(a)}\right|}$. Thus $\mathcal{Z}(\infty)$ is

$$
Z_{\alpha^{(a)}} \cap \mathcal{H}_{\alpha^{(a)}}=\bigcup_{\beta^{(b)} \lessdot \alpha^{(a)}} Z_{\beta^{(b)}},
$$

by Proposition 2.1 (ii).

Claim: The cycle

$$
\mathcal{Z}(\infty) \cap \mathcal{H}\left(s_{1}, 1\right) \cap \cdots \cap \mathcal{H}\left(s_{k}, 1\right)
$$

is free of multiplicities.

If not, then there are two components $Z_{\beta^{(b)}}$ and $Z_{\gamma^{(c)}}$ of $\mathcal{Z}(\infty)$ such that

$$
Z_{\beta^{(b)}} \cap Z_{\gamma^{(c)}} \cap \mathcal{H}\left(s_{1}, 1\right) \cap \cdots \cap \mathcal{H}\left(s_{k}, 1\right)
$$

is non-empty. But this contradicts Theorem 3.2 as $Z_{\beta^{(b)}} \cap Z_{\gamma^{(c)}}=Z_{\delta^{(d)}}$, where $\delta^{(d)}$ is the greatest lower bound of $\beta^{(b)}$ and $\gamma^{(c)}$ in $\mathcal{C}_{q}$, and so $\operatorname{dim} Z_{\delta^{(d)}}<\operatorname{dim} Z_{\beta^{(b)}}=k$.

From the claim, there is a real number $\mathcal{N}_{\alpha^{(a)}}>0$ such that if $s>\mathcal{N}_{\alpha^{(a)}}$, then

$$
\mathcal{Z}(s) \cap \mathcal{H}\left(s_{1}, 1\right) \cap \cdots \cap \mathcal{H}\left(s_{k}, 1\right)
$$

is transverse with all points of intersection real. Set

$$
\mathcal{N}_{k+1}:=\max \left\{\mathcal{N}_{\alpha^{(a)}}:\left|\alpha^{(a)}\right|=k+1\right\}
$$

and let $s_{k+1}$ be any real number satisfying $s_{k+1}>\mathcal{N}_{k+1}$. 


\section{FURTHER REMARKS}

The proof of Theorem 3.1 gives a rather precise choice of $s_{i}$ and $L_{i}$ in the enumerative problem (1.1) which gives only real maps. The positive real numbers $s_{1}, \ldots, s_{N}$ of Theorem 3.1 are constructed inductively, first choosing $s_{1}>0$. Then, having chosen $s_{1}, \ldots, s_{k}$, a number $\mathcal{N}_{k+1}>0$ is found with the property that for any $s_{k+1}>\mathcal{N}_{k+1}$ and $\alpha^{(a)}$ with $\left|\alpha^{(a)}\right|=k+1$, the intersection (3.1) is transverse with all points real. By the quantifier $\forall s_{1} \ll s_{2} \ll \cdots \ll s_{N}$, we mean such a choice of real numbers $s_{1}, \ldots, s_{N}$. More precisely,

$$
\forall s_{1}>0 \quad \exists \mathcal{N}_{2}>0 \text {, such that } \forall s_{2}>\mathcal{N}_{2} \quad \cdots \quad \exists \mathcal{N}_{N}>0 \text {, such that } \forall s_{N}>\mathcal{N}_{N} .
$$

We deduce a more precise form of Theorem 1.1, as the quantifiers $\forall s_{1} \ll s_{2} \ll$ $\cdots \ll s_{N}$ and $\forall s_{1}^{n} \ll s_{2}^{n} \ll \cdots \ll s_{N}^{n}$ are equivalent.

Corollary 4.1. $\forall s_{1} \ll s_{2} \ll \cdots \ll s_{N}$, each of the d maps $M: \mathbb{P}^{1} \rightarrow \mathbf{G}$ of degree $q$ which satisfy $M\left(s_{i}, 1\right) \cap L\left(s_{i}^{1 / n}, 1\right) \neq\{0\}$ for $i=1, \ldots, N$ are real.

When $q=0$, there is substantial evidence [16] that this choice of $s_{1}, \ldots, s_{N}$ is too restrictive. B. Shapiro and M. Shapiro have the following conjecture:

Conjecture 4.2 (B. Shapiro and M. Shapiro). Suppose $q=0$. Then for distinct real numbers $s_{1}, \ldots, s_{m p}$ each of the finitely many p-planes $H$ which satisfy $H \cap$ $L\left(s_{i}, 1\right) \neq\{0\}$ are real.

In contrast, when $q>0$ the restriction $\forall s_{1} \ll s_{2} \ll \cdots \ll s_{N}$ is necessary. We observe this in the case of $q=1, p=m=2$, so $N=8$ and $d=8$; that is, for parameterized curves of degree 1 in the Grassmannian of 2-planes in $\mathbb{C}^{4}$. Here, the choice of $s_{i}=i$ in (3.3) gives no real maps, while the choice $s_{i}=i^{6}$ gives 8 real maps.

We describe that calculation. There are 12 Plücker coordinates $z_{i j(a)}$ for $1 \leq i<$ $j \leq 4$ and $a=0,1$. If we let $f_{i j}:=t z_{i j^{(0)}}+s z_{i j^{(1)}}$, then

$$
f_{14} f_{23}-f_{13} f_{24}+f_{12} f_{34}=0,
$$

as $f_{i j}(s, t) \in \mathbf{G}$ for all $s, t$. The coefficients of $t^{2}, s t$, and $s^{2}$ in this expression give three quadratic relations among the $z_{i j(a)}$ :

$$
\begin{gathered}
z_{14^{(0)}} z_{23^{(0)}}-z_{13^{(0)}} z_{24^{(0)}}+z_{12^{(0)}} z_{34^{(0)}}, \\
z_{12^{(1)}} z_{34^{(0)}}-z_{13^{(1)}} z_{24^{(0)}}+z_{14^{(1)}} z_{23^{(0)}}+z_{23^{(1)}} z_{14^{(0)}}-z_{24^{(1)}} z_{13^{(0)}}+z_{34^{(1)}} z_{12^{(0)}}, \\
z_{14^{(1)}} z_{23^{(1)}}-z_{13^{(1)}} z_{24^{(1)}}+z_{12^{(1)}} z_{34^{(1)}},
\end{gathered}
$$

and these constitute a Gröbner basis for the homogeneous ideal of $\mathcal{K}_{1}$ [18.

Here, the form $\Lambda(s, 1)$ is

$$
\begin{gathered}
z_{12^{(0)}}-2 s z_{13^{(0)}}+s^{2} z_{14^{(0)}}+3 s^{2} z_{23^{(0)}}-2 s^{3} z_{24^{(0)}}+s^{4} z_{34^{(0)}} \\
+s^{4} z_{12^{(1)}}-2 s^{5} z_{13^{(1)}}+s^{6} z_{14^{(1)}}+3 s^{6} z_{23^{(1)}}-2 s^{7} z_{24^{(1)}}+s^{8} z_{34^{(1)}} .
\end{gathered}
$$

We set $z_{34^{(1)}}=1$ and work in local coordinates. Then the ideal generated by the 3 quadratic equations and 8 linear relations $\Lambda\left(s_{i}, 1\right)$ for $i=1, \ldots, 8$ defines the 8 solutions to (3.3). We used Maple to generate these equations and compute the number of real solutions. There are no real solutions when $s_{i}=i$, but all 8 are real when $s_{i}=i^{6}$. 
We describe how the enumerative problem (1.1) arises in systems theory (see also [3]). A physical system (e.g. a mechanical linkage) with $m$ inputs and $p$ measured outputs whose evolution is governed by a system of linear differential equations is modeled by an $m \times n$-matrix $L(s)$ of real univariate polynomials. The largest degree of a maximal minor of this matrix is the McMillan degree, $r$, of the evolution equation. Consider now controlling this linear system by output feedback with a dynamic compensator. That is, use a $p$-input, $m$-output linear system $M$ to couple the $m$ inputs of the system $L$ to its $p$ outputs. The resulting closed system has characteristic polynomial

$$
\varphi(s):=\left[\begin{array}{c}
L(s) \\
M(s)
\end{array}\right],
$$

and the roots of $\varphi$ are the natural frequencies or poles of the closed system. The dynamic pole assignment problem asks, given a system $L(s)$ and a desired characteristic polynomial $\varphi$, can one find a (real) compensator $M(s)$ of McMillan degree $q$ so that the resulting closed system has characteristic polynomial $\varphi$ ? That is, if $s_{1}, \ldots, s_{r+q}$ are the roots of $\varphi$, then which $M \in \mathcal{M}_{q}$ satisfy

$$
\operatorname{det}\left[\begin{array}{c}
L\left(s_{i}\right) \\
M\left(s_{i}\right)
\end{array}\right]=0, \quad \text { for } i=1,2, \ldots, r+q ?
$$

In the critical case when $r+q=m p+q n\left(=\operatorname{dim} \mathcal{M}_{q}\right)$, this is an instance of the enumerative problem (1.1). When the degree $d$ is odd, then for any real system $L$ and a real characteristic polynomial $\varphi$, there will be at least one real dynamic compensator. Part of the motivation for [11] was to obtain a closed formula for $d$ from which its parity could be deduced for different values of $q, m$, and $p$.

The choice of planes $L_{i}$ that arise in the dynamic pole placement problem are $N=m p+q n$ points on a rational curve of degree $m p+(n-1) q$ in the Grassmannian of $m$-planes in $\mathbb{C}^{n}$. In contrast, the planes of Theorem 3.1 (and hence of Theorem 1.1) arise as $N$ points on a rational curve of degree $m p$. Only when $q=0$ (the case of static compensators) is there overlap.

Our proof of Theorem 1.1 (like that in [17) was inspired by the numerical Pieri homotopy algorithm of 7 ] for computing the solutions to (1.1) when $q=0$. Likewise, our explicit degenerations of intersections of the $\mathcal{H}(s, t)$, and more generally Proposition 2.1 (ii), suggested to Huber and Verschelde an optimal numerical homotopy algorithm for finding the solutions to (1.1) 6]. This is in exactly the same manner as the explicit degenerations of intersections of special Schubert varieties of [15] were used to construct the Pieri homotopy algorithm of [7] (see also [6]).

We close with one open problem concerning the enumeration of rational curves on a Grassmannian. For a point $s \in \mathbb{P}^{1}$ and a Schubert variety $\Omega$ of $\mathbf{G}$, consider the quantum Schubert variety $\Omega(s)$ of curves $M \in \mathcal{M}_{q}$ satisfying $M(s) \in \Omega$. Bertram's quantum Schubert calculus gives formulas to compute the number of curves $M \in$ $\mathcal{M}_{q}$ which lie in the intersection of an appropriate number of these $\Omega(s)$, and we ask when it is possible to have all solutions real. A modification of the proof of Theorem 3.1] shows that this is the case when all except possibly 2 are hypersurface Schubert varieties. In every case we have computed, all solutions may be real.

\section{ACKNOWLEDGEMENT}

We thank Linda Chen, who helped us better understand the relation between the varieties $\mathcal{Q}_{q}$ and $\mathcal{K}_{q}$. 


\section{REFERENCES}

[1] A. Bertram, Quantum Schubert calculus, Adv. Math. 128 (1997), no. 2, 289-305. MR 98j:14067

[2] A. Bertram, G. Daskalopoulos, and R. Wentworth, Gromov invariants for holomorphic maps from Riemann surfaces to Grassmannians, J. Amer. Math. Soc. 9 (1996), no. 2, 529-571. MR 96f:14066

[3] C. I. Byrnes, Pole assignment by output feedback, Three Decades of Mathematical Systems Theory (H. Nijmeijer and J. M. Schumacher, eds.), Lecture Notes in Control and Inform. Sci., vol. 135, Springer-Verlag, Berlin, 1989, pp. 31-78. MR 90k:93001

[4] J. M. Clark, The consistent selection of local coordinates in linear system identification, Proc. Joint Automatic Control Conference, 1976, pp. 576-580.

[5] Wm. Fulton, Introduction to intersection theory in algebraic geometry, CBMS 54, AMS, 1984. MR 85j:14008

[6] B. Huber and J. Verschelde, Pieri homotopies for problems in enumerative geometry applied to pole placement in linear systems control, MSRI 1999-028, SIAM J. Control and Optim., to appear.

[7] B. Huber, F. Sottile, and B. Sturmfels, Numerical Schubert calculus, J. Symb. Comp. 26 (1998), no. 6, 767-788. CMP 99:06

[8] K. Intriligator, Fusion residues, Mod. Phys. Lett. A 6 (1991), 3543-3556. MR 92k:81180

[9] S. Kleiman, The transversality of a general translate, Compositio Math. 28 (1974), 287-297. MR 50:13063

[10] M. S. Ravi and J. Rosenthal, A smooth compactification of the space of transfer functions with fixed McMillan degree, Acta Appl. Math. 34 (1994), 329-352. MR 95b:93043

[11] M. S. Ravi, J. Rosenthal, and X.C. Wang, Dynamic pole assignment and Schubert calculus, SIAM J. Control and Optim. 34 (1996), 813-832. MR 97c:93051

[12] Degree of the generalized Plücker embedding of a quot scheme and quantum cohomology, Math. Ann. 311 (1998), no. 1, 11-26. CMP 98:13

[13] F. Ronga, A. Tognoli, and Th. Vust, The number of conics tangent to 5 given conics: the real case, Rev. Mat. Univ. Complut. Madrid 10 (1997), 391-421. MR 99d:14059

[14] J. Rosenthal, On dynamic feedback compensation and compactification of systems, SIAM J. Control Optim. 32 (1994), 279-296. MR 95b:93084

[15] F. Sottile, Pieri's formula via explicit rational equivalence, Can. J. Math. 49 (1997), no. 6, 1281-1298. CMP 98:09

[16] _ Real Schubert calculus: Polynomial systems and a conjecture of Shapiro and Shapiro, 28 pp., Exper. Math., to appear. 1999.

[17] $\longrightarrow$ The special Schubert calculus is real, ERA of the AMS 5 (1999), 35-39. CMP 99:11

[18] F. Sottile and B. Sturmfels, A sagbi basis for the quantum Grassmannian, 16 pages, 1999. math. AG/9908016

[19] S. A. Strømme, On parameterized rational curves in Grassmann varieties, Space Curves (F. Ghione, C. Peskine, and E. Sernesi, eds.), Lecture Notes in Math., vol. 1266, SpringerVerlag, 1987, pp. 251-272. MR 88i:14020

[20] C. Vafa, Topological mirrors and quantum rings, Essays on Mirror Manifolds, International Press, 1992, ed. by S.-T. Yau, pp. 96-119. MR 94c:81193

Department of Mathematics, University of Wisconsin, Van Vleck Hall, 480 Lincoln Drive, Madison, Wisconsin 53706-1388

Current address: Department of Mathematics, University of Massachusetts, Amherst, Massachusetts 01003-4515

E-mail address: sottile@math.umass.edu

URL: http://www . math. umass.edu/ sottile 